\title{
Esterilización quirúrgica femenina con anestesia local en el Instituto Materno Infantil (IMI) de Santafé de Bogotá, D.C.
}

\author{
Pío Iván Gómez Sánchez*; Jaime Fernando Ruiz Povea**; Mario Orlando Parra***; Liliana Arango****; Carlos \\ Espitia*****; Cristina González******
}

RESUMEN: La esterilización quirúrgica femenina es el método anticonceptivo más utilizado en Colombia y el entrenamiento a los médicos en técnicas sencillas y seguras, debe ser una prioridad en las escuelas médicas de un país que como el nuestro, la planificación familiar es una necesidad sentida de la población.

En el Instituto Materno Infantil de Santafé de Bogotá, institución de tercer nivel que atiende pacientes con alto riesgo reproductivo se ha adquirido experiencia en la implementación de la esterilización tubárica por minilaparotomía utilizando anestesia local (técnica que se describe), con facilidades en el entrenamiento del personal y disminución de costos hospitalarios.

El presente es un estudio descriptivo, retrospectivo en el cual se revisan registros de 878 pacientes a quienes se realizó la técnica mencionada entre el 1 de enero de 1990 y el 31 de Diciembre de 1996. En 55\% de los casos se realizó la ligadura postparto (menos de 48 horas postalumbramiento). La edad promedio fue de 31 años y el peso promedio de $60 \mathrm{Kg}$ (rango: $37-92 \mathrm{Kg}$ ). Tan solo en el $16 \%$ de las pacientes se requirió anestesia general. Hubo complicaciones en el $2.8 \%$ de los casos. Se han presentado 2 fallas hasta el momento.

En todos los casos el procedimiento fue ambulatorio.

Se concluye que la técnica de esterilización femenina por minilaparotomía realizada con anestesia local es un método barato y seguro de anticoncepción permanente que adecuadamente implementado puede contribuir a disminuir las gestaciones de alto riesgo y a mejorar la calidad de vida en muchas áreas del país.

PALABRAS CLAVES: Esterilización femenina, anestesia local.

SUMMARY: Female surgycal sterilization is the most used contraceptive method in Colombia, and safe and simple techniques must be considered in general and specialized medical training programmes. At Instituto Materno Infantil in Santafé de Bogotá, experience has been adquiered in tubal ligation performing a minilaparotomy under local anaesthesia. With notorious advantages in new personnel training and reducing institutional costs.

This study is a descriptive retrospective one, in wich records of 878 patients who undergone tubal ligation were reviewed, since January 1 st until December 31st 1996.

$55 \%$ of tubal ligations were performed in the postpartum period. Mean patients age was 31 years and mean weight was $60 \mathrm{Kg}$ (rank 37 92). In $16 \%$ of cases it was necesary complete the procedure under general anaesthesia. Intraoperatory complications were present in $2.8 \%$ of procedures. There have been 2 methods failures. In all cases the procedure was performed ambulatory.

We concluded tubal ligation performing a minilaparotomy under local anaesthesia is a safe, cost-effective contraceptive method wich might enhance life quality in many Colombian areas.

KEY WORDS: Female sterilization, local anaesthesia.

\section{Introducción}

El Instituto Materno Infantil de Santafé de Bogotá D.C. (IMI), es una institución que atiende pacientes de

\footnotetext{
* Profesor Asociado. Director del Departamento de Ginecología y Obstetricia . Universidad Nacional de Colombia. Asesor Unidad Planificación Familiar IMI.

** Residente II Ginecobstetricia. Universidad Nacional de Colombia. Unidad Planificación Familiar IMI.

*** Instructor Asociado. Dpto. Ginecología y Obstetricia. Universidad Nacional de Colombia. Unidad Planificación Familiar IMI.

**** Ginecobstetra. Cirugía Ambulatoria IMI.

***** Anestesiólogo. Unidad Cirugía Ambulatoria IMI.

****** Profesional Enfermería. Unidad Planificación Familiar IMI.
}

alto riesgo y una de las principales formas de disminuir la morbimortalidad materna es proporcionando métodos anticonceptivos que permitan a las usuarias decidir libremente sobre el ejercicio de la reproducción, de tener hijos o no, cuantos y en que momento (1), además el IMI tiene la responsabilidad de ofrecer información y servicios al respecto indicando ventajas, desventajas y riesgos de la reproducción en circunstancias personales y familiares determinadas (2-3).

Hasta 1989 el IMI remitía sus pacientes para planificación al Centro de Salud No. 16 de la Secretaría de Salud de Bogotá, el cual no cubría tal demanda. A comienzos de 1990 con el apoyo de la Association for Voluntary Surgycal Contraception (AVSC), con sede en Estados 
Unidos y con una Oficina Regional en Bogotá, se inicia la Unidad de Planificación Familiar del IMI con el fin de brindar un servicio integral que ofrezca todos los métodos disponibles, tanto reversibles como irreversibles, de tal forma que la mujer o la pareja puedan decidir libremente el método que deseen, luego de una completa consejería por parte del personal de salud de la Unidad, para tal fin se cuenta con audiovisuales, rotafolios y volantes ilustrativos. Existe una enfermera jefe que da consejería a pacientes hospitalizadas y en consulta externa, además brinda entrenamiento al personal auxiliar.

Se proporcionan en esta Unidad: métodos de barrera, anovulatorios orales, inyectables mensuales y trimestrales, implantes subdérmicos y anticoncepción quirúrgica voluntaria (AQV), tanto femenina como masculina, realizadas con anestesia local con amplias ventajas para los pacientes y bajos costos para el IMI. El método lo escoge la pareja o la mujer cuando asiste sola, después del proceso de consejería. No prescribimos métodos, solo brindamos la opción de escoger el que los usuarios deseen, de acuerdo a sus necesidades y riesgo reproductivo específico.

La AQV en la mujer es uno de los métodos más utilizados en el mundo existiendo cerca de 100 millones de mujeres con esterilización quirúrgica. En Colombia es el primer método en uso, especialmente en algunas zonas como la costa Atlántica (4).

De las técnicas quirúrgicas realizadas por minilaparotomía, en nuestro medio la técnica de Pomeroy (data de 1930), Uchida (data de 1935) y Parkland son las más difundidas. De las técnicas con Laparoscopia, la electrocoagulación unipolar ya no se usa pues dañaba entre el $20-50 \%$ de la trompa, en algunos sitios se usa la electrocoagulación bipolar y termocoagulación que dañan menos trompas pero en nuestro medio, lo más utilizado especialmente en los programas privados son los anillos tubáricos, desarrollados por el Dr. In Bae Yoon en 1973, utilizando el Laparoscopio Laprocator, menos costoso y menos difícil de mantener que otros laparoscopios, el anillo destruye entre uno y tres centímetros de trompa (5).

Otro método oclusivo es el uso de ganchos, que dañan incluso menos porción de trompa que el anillo de Yoon, existen varios como el desarrollado por Hulka en 1972, dotado de resorte, el desarrollado por Filshie en 1973 hecho de titanio y revestido internamente por silicón. Otros como el Hemoclip de tantalium y el de Bleier, han sido retirados por la alta tasa de embarazos, sin embargo, en nuestro medio los ganchos son pocos utilizados en general.

En nuestra institución la técnica más utilizada es la minilaparotomía con anestesia local y materiales absorvibles, tanto en intervalo como en postparto, sin embargo, cuando esta técnica está contraindicada, usamos con frecuencia la esterilización laparoscópica con coagulación bipolar pero con una variación en la cual no solo se coagula y corta la trompa, sino que resecamos un segmento tubárico de 0.5 a $1.0 \mathrm{cms}$, lo cual demora pocos minutos más la cirugía pero da una mejor seguridad. Rara vez usamos bandas de silástic, por su alto costo, poca disponibilidad y mayor tasa de fracaso. En esta publica- ción nos referiremos a la esterilización realizada con minilaparotomía bajo anestesia local.

\section{Materiales y métodos}

El presente es un estudio descriptivo retrospectivo, cuyo universo lo comprende las pacientes atendidas en la Unidad de Planificación Familiar del IMI durante el lapso comprendido entre el 1 de enero de 1990 y el 31 de Diciembre de 1996 y a quienes se les realizó esterilización quirúrgica con minilaparatomía y anestesia local en la Unidad de Planificación Familiar del IMI.

Se tomaron como fuente de datos: los libros de registro de la Unidad, la historia institucional y la historia precodificada que se utiliza para tal fin.

El objetivo de este trabajo es mostrar la experiencia con esterilización quirúrgica femenina realizada con anestesia local. Como pre-requisito es necesario que la mujer haya recibido amplia y suficiente consejería en la cual se explica la técnica y se induce a que practique el movimiento abdominal requerido en el procedimiento, para ascender las estructuras intestinales y facilitar el acceso a las trompas.

Esta técnica no debe realizarse en mujeres con obesidad marcada, con antecedente de intervenciones abdominales bajas, sospecha de adherencias tubo-ováricas o cuando el cirujano no está entrenado en la técnica de AQV con anestesia local.

Esta cirugía se puede realizar en el postparto inmediato o hasta las 48 horas con una incisión infraumbilical, también se puede realizar en intervalo usando cánula uterina y con incisión suprapúbica.

Los pasos de la técnica son:

1. Consejería y selección de las pacientes.

2. Lavado quirúrgico del área. Colocación de cánula uterina si es de intervalo, no se debe colocar si es en postparto. La paciente estará en posición ginecológica si la cirugía es de intervalo y en decúbito supino si es de postparto.

3. Infiltración con xilocaína, en el postparto la incisión será en el borde inferior del ombligo, en intervalo será suprapúbica. La infiltración se hace con xilocaína sin epinefrina, diluida al $0.5 \%$, iniciando muy superficialmente en la piel (lograr piel de naranja), luego se infiltra en un ángulo de 45 grados el tejjido celular subcutáneo hacia arriba, abajo, derecha e izquierda, siempre aspirando la jeringa para evitar inyecciones intravasculares. No usar mucho volumen de anestésico.

4. Incisión en piel con bisturí con extensión entre 2$4 \mathrm{cms}$.

5. Disección de grasa al introducir separadores de piel e infiltración anestésica de aponeurosis y músculo. Usar poco volumen.

6. Identificación de peritoneo e infiltración si es necesaria. Apertura con técnica habitual.

7. Maniobra de ascenso intestinal por parte de la paciente, con movimientos respiratorios. (Enseñada con anterioridad en la Consejería, muchas pacientes comprenden con términos como: "Hágase flaca", "meta el estómago", "recuerde como hace cuando se coloca un pantalón ajustado.") 
8. Identificación de trompa derecha con manipulador de trompa. Se usa no para extraer la trompa sino para desplazarla hacia el centro y extraerla con una pinza de disección atraumática o pinza de babcock si cabe por la incisión. A veces es necesario gotear xilocaína en la cavidad abdominal.

El manipulador de la trompa es un instrumento muy sencillo que consiste en una varilla de $2-3 \mathrm{~mm}$ de grosor y que termina con un pequeño círculo en ángulo recto en la punta, se puede reemplazar por una pinza de cístico delgada. (Ver Figuras 1 y 2 )

9. Realización de la esterilización, en el IMI generalmente utilizamos la técnica de Parkland. (Siempre materiales de sutura absorvibles)

10. Se repite procedimiento en lado izquierdo.

11. Revisión de hemostasia.

12. Cierre por planos. Cuando la incisión en aponeurosis es menor de $2 \mathrm{cms}$, se puede suturar con catgut crómico, uno o dos puntos separados y con el

Figura 1

\section{MANIPULADOR DE TROMPA}

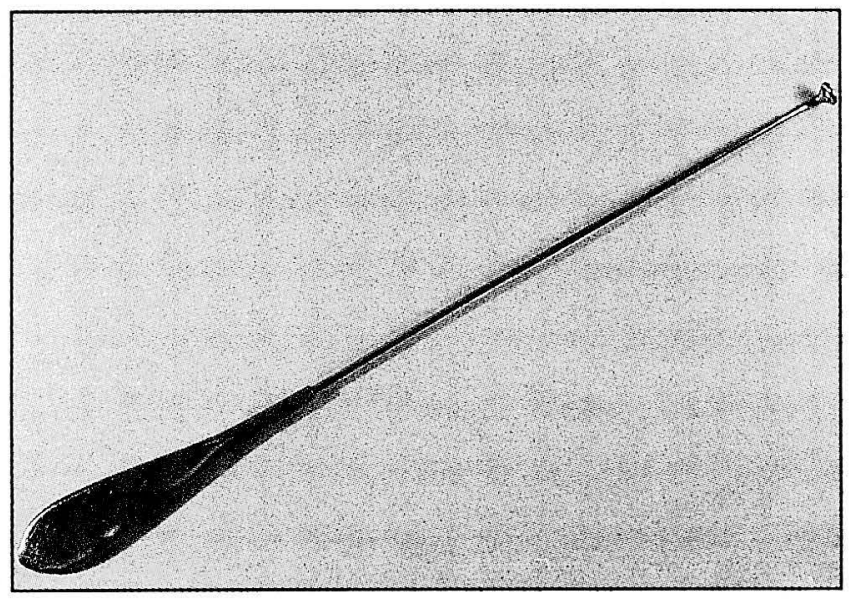

Figura 2

DETALLE MANIPULADOR DE TROMPA

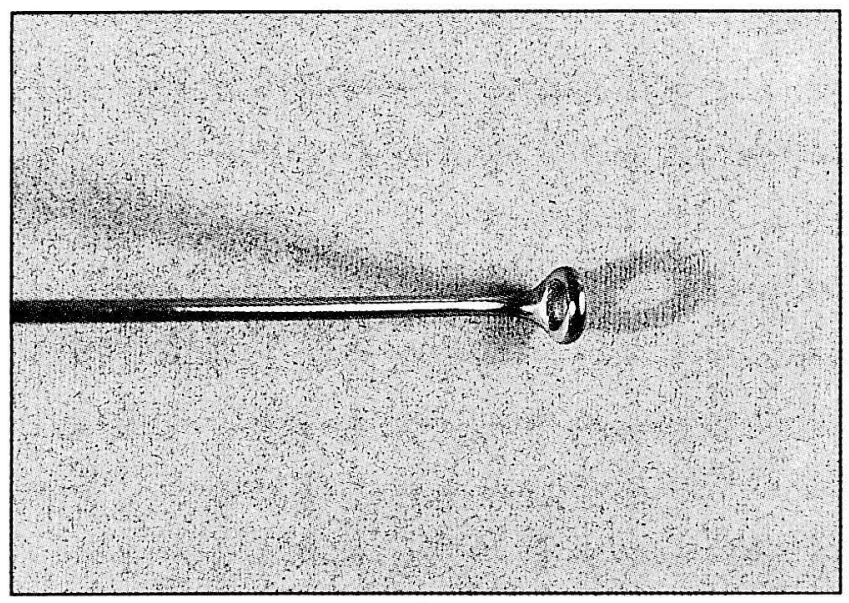

mismo material colocamos un punto invaginante en piel. Cuando la incisión inicial fue en aponeurosis mayor de 2 cms realizamos la cirugía tubárica y el cierre de pared con material de absorción lento.

Debemos insistir en que el manejo de los tejidos por parte del cirujano debe ser muy cuidadoso, no se deben realizar maniobras bruscas ni lastimar estructuras que no están anestesiadas, durante el procedimiento se debe hablar con la paciente para disminuirle la tensión. En el IMI aprovechando el monitor que se utiliza en Videolaparoscopia, usamos un video de relajación antes y durante la cirugía.

En algunos casos es necesario sedar la paciente y se utilizan dosis bajas de Midazolam con Fentanyl. En un hospital universitario como el nuestro existe el recurso de anestesiólogo disponible, pero realmente para esta técnica no es indispensable.

\section{Elección de paciente}

\section{Elección voluntaria}

Autorización escrita previo conocimiento de implicaciones y repercusiones.

No existe en el país limitante legal de edad o paridad para la cirugía, en el caso de la mujer mayor de edad solamente se requiere adecuada consejería y la firma del consentimiento informado, el cual no requiere la firma del compañero de la paciente ni la firma de testigos, solamente si la mujer es analfabeta, se necesita un testigo para certificar que el texto del consentimiento informado que se le explicó a la paciente es el mismo donde pone la paciente su huella digital. (Ver formato en la página siguiente)

En la mujer menor o mayor de edad donde se requiera $A Q V$ por enfermedades mentales que afecten su maternidad debemos hacer valoración psiquiátrica que nos aclare el verdadero diagnóstico del estado mental y poder definir si hay o no limitación para cumplir su rol de madre, si hay concepto psiquiátrico a favor de la esterilización quirúrgica, puede llevarse a cabo la cirugía luego de que un juez ratifique la condición de inimputabilidad de la mujer y asigne los tutores quienes serán los que autoricen la cirugía.

\section{Resultados}

Se revisan en este período estudiado 878 pacientes a quienes se les realizó esterilización tubaria: 488 en postparto, 369 de intervalo y 21 en postaborto, (ver figura 3 ). Cerca del $62 \%$ fueron realizadas por personal en entrenamiento, tanto de pregrado como de postgrado. La edad de las pacientes estuvo en un rango de 20 a 47 años, con un promedio de 31 años, el peso de las mismas estuvo entre 37 y $92 \mathrm{Kg}$ con promedio de $60 \mathrm{Kg}$.

Del grupo total de pacientes llevadas a AQV con la técnica de anestesia local en el $16 \%$ hubo necesidad de suministrarles anestesia general o sedación profunda, por dificultades técnicas (adherencias intrapélvicas), incomodidad de la paciente o complicaciones.

La cantidad promedio de xilocaína al $0.5 \%$ sin epinefrina utilizada para todo el procedimiento fue me- 
Figura 3

\section{AQV CON ANESTESIA LOCAL MOMENTO DE REALIZACION}

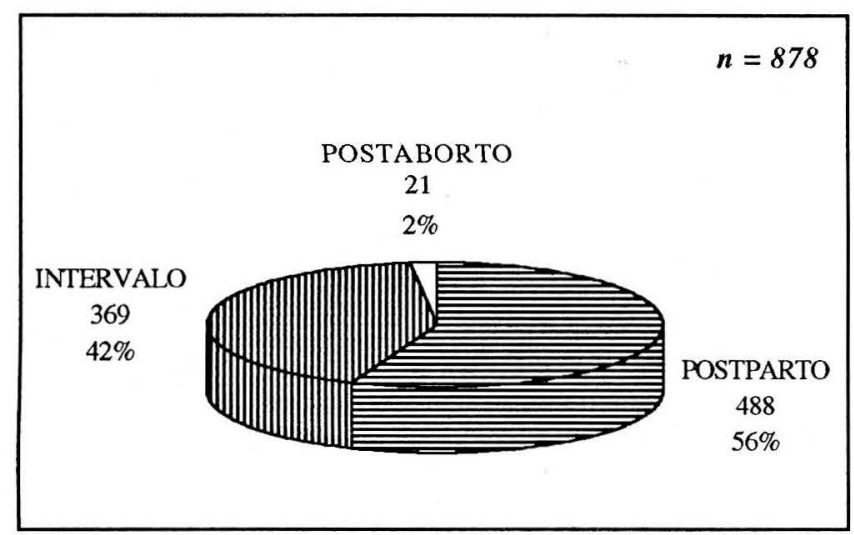

nor de $240 \mathrm{mg}$ en el $89 \%$ de los casos, en más del $95 \%$ de los casos sólo se utilizó una sutura absorvible, para todo el procedimiento quirúrgico.

El promedio de tiempo de cirugía fue menor de 25 minutos en cerca del $86 \%$, con tiempos entre 6 minutos y 40 minutos, estas últimas en los casos de entrenamiento a estudiantes de pregrado.

El tamaño de la incisión estuvo entre 1.5 a $4 \mathrm{cms}$, siendo en el $90 \%$ de los casos menor de $3 \mathrm{cms}$.

El tipo de técnica más utilizada fue la técnica de Parkland en el $80 \%$ de los casos, seguida de Pomeroy modificado en el $12 \%$ y Uchida en el $2 \%$

Complicaciones del procedimiento se presentaron en el $2.8 \%$, siendo la principal el desgarro del mesosalpinx, el cual es evitable con la cuidadosa manipulación de los tejidos, que se adquiere con el entrenamiento. Dentro de este porcentaje se incluyen 4 casos de infección de la herida quirúrgica, 2 casos de lesiones vesicales, 2 de

\section{INSTITUTO MATERNO INFANTIL DE SANTAFE DE BOGOTA D.C UNIDAD DE PLANIFICACION FAMILIAR \\ UNIVERSIDAD NACIONAL DE COLOMBIA}

\section{SOLICITUD DE ATENCION MEDICA PARA ANTICONCEPCION QUIRURGICA VOLUNTARIA (LIGADURA DE TROMPAS O VASECTOMIA)}

HISTORIA CLINICA No.

FECHA...........de 199...

Yo, ..de.......años y con...... hijos, en pleno uso de mis facultades mentales y habiendo sido informado(a) y comprendiendo la existencia de los diferentes métodos anticonceptivos disponibles, solicito y autorizo voluntariamente al Instituto Materno Infantil de Santafé de Bogotá D.C, para que el médico tratante me efectúe el procedimiento (ligadura de trompas en la mujer o vasectomía en el hombre).

Se me ha explicado y comprendo lo siguiente:

1. Que hay métodos anticonceptivos temporales que puedo utilizar en lugar de la cirugía para planificar la familia.

2. Que el procedimiento seleccionado es quirúrgico y el personal del Instituto me ha explicado los detalles.

3. Que la intervención quirúrgica tiene riesgos, los cuales me han sido explicados.

4. Que si la operación tiene éxito, no podré tener más hijos.

5. Que los efectos de la operación son permanentes e irreversibles.

6. Que puedo cambiar de opinión en cualquier momento y decidir no operarme, sin que por ello pierda el derecho a recibir los servicios y la atención médica del Instituto.

Esta determinación ha sido tomada libre y voluntariamente, sin coacción ni aliciente alguno y luego de consultar con mi pareja, para constancia firmo esta SOLICITUD Y AUTORIZACION.

FIRMA Y HUELLA DACTILAR DEL USUARIO(A) 
lesiones intestinales y 3 de perforación uterina con la cánula de manipulación uterina. Todas las complicaciones fueron tratadas por el mismo cirujano y cabe anotar que las complicaciones intestinales, vesicales y perforaciones uterinas no fueron causadas por médicos internos sino por especialistas o médicos residentes, de otra parte las complicaciones menos serias como los desgarros de mesosalpinx, fueron causadas por médicos internos. (Ver Figuras 4 y 5)

Hasta el momento solo tenemos 2 casos de falla del método y ambos han correspondido a técnica de Pomeroy modificado, ninguno a Parkland o Uchida.

Dentro de los hallazgos operatorios insospechados tuvimos un quiste de paraovario de $5 \mathrm{cms}$ de diámetro y una gestación ectópica tubárica no rota, cuyo tratamiento se realizo en el mismo acto operatorio y con anestesia local.

La estancia hospitalaria postcirugía no sobrepaso las 2 horas, siendo realmente un procedimiento ambulatorio.

Figura 4

\section{COMPLICACIONES AQV -}

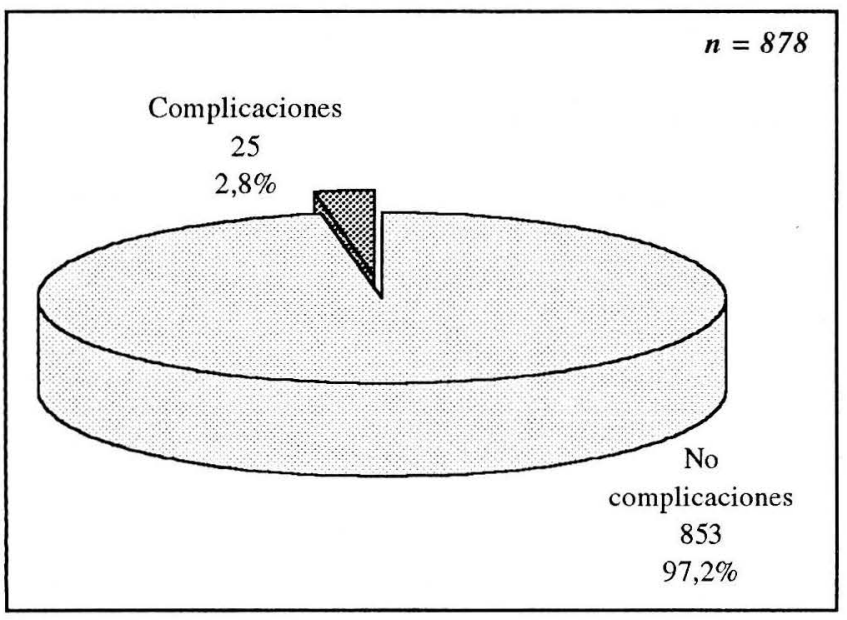

Figura 5

COMPLICACIONES AQV

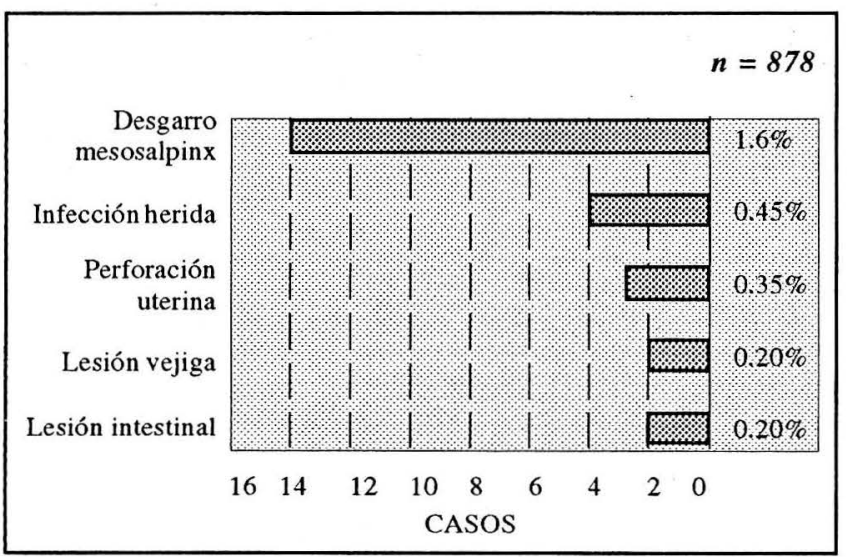

\section{Discusión}

La AQV debe procurarse realizar con materiales de sutura absorvibles, porque con materiales inabsorvibles (seda por ejemplo), hay mayor riesgo de recanalización tubaria (6).

Cualquiera que sea la técnica usada debe ser hecha a nivel ístmico pues allí el calibre tubario es uniforme y si en el futuro hay necesidad de recanalización es técnicamente más sencilla (7).

La pregunta es: cuál de los métodos de oclusión tubárica es más confiable, la respuesta no es fácil pues faltan estudios comparativos a largo plazo.

A finales de 1996 se conoció el resultado de un gran estudio multicéntrico prospectivo realizado por el "Collaborative Review of Sterilization" en USA (8), el cual confirmó que la oclusión tubárica es un método de anticoncepción altamente eficaz, pero nos llama la atención a que la esterilización tubárica conlleva un riesgo de embarazo tardío especialmente con algunas técnicas. De las 10865 mujeres, el seguimiento fue de $89.2 \%$ para el primer año, $81 \%$ para tres años, $73 \%$ para 5 años y $57.7 \%$ entre 8-14 años. La edad media de esterilización fue de 30 años y la mayoría habían tenido por lo menos 2 gestaciones, se hallaron 143 gestaciones clasificadas como fallas de esterilización, el 32.9\%(47 casos) fueron ectópicas, $28.7 \%$ (41 casos) terminaron en parto, $18.2 \%$ (26 casos) en aborto inducido y $14.7 \%(21$ casos) en aborto espontáneo.

La probabilidad acumulativa de embarazo de 10 -años por mil esterilizaciones tubáricas fue más alta para la aplicación de clips, elevándose a 36.5, luego en orden descendente, siguieron la coagulación bipolar con 24.8, salpinguectomía parcial de intervalo con 20.1, bandas de silástic con 17.7, coagulación monopolar con 7.5 y salpinguectomía parcial postparto con 7.5.

Estos hallazgos deben interpretarse con detenimiento y sumo cuidado, debemos concluir que las dos terceras partes de gestaciones fueron ectópicas, por lo cual este antecedente debe tenerse en mente siempre. El hecho de que la coagulación monopolar mostró baja tasa de fracaso no debe animarnos a realizarla pues los potenciales riesgos de daño a órganos vecinos nos ha enseñado a restringir su uso.

La creación de la Unidad de Planificación Familiar del IMI ha tenido un resultado muy positivo para la comunidad, el cual no solo ha contribuido a mejorar las condiciones de bienestar de la familia a través de la prevención con educación, atención integral de los grupos de alto riesgo como las adolescentes, sino que ha despertado concientización frente a la responsabilidad del embarazo y las enfermedades transmitidas sexualmente, así como la preocupación por la detección precoz del cáncer de seno y cérvix.

En el programa de esterilización quirúrgica femenina con anestesia local se ha dado la prioridad de entrenamiento a los médicos que están terminando su formación (médicos internos) y a Residentes, pero se han entrenado médicos de varias regiones del país e inclusive del extranjero. Al ser una técnica que utiliza recursos mínimos, y anestesia local, se puede realizar en cualquier zona del 
país, especialmente en zonas rurales, donde la carencia de programas de planificación o la falta de anestesiólogos han limitado el acceso a la AQV y es allí precisamente, donde la tasa global de fecundidad no ha tenido cambios importantes, como si ha sucedido en ciudades capitales del país. Con la anterior motivación estamos entrenando a nuestros estudiantes de pre y postgrado, para difundir esta técnica y no tener la necesidad, como hasta ahora, de que regresen a nuestra institución desde zonas apartadas del país para entrenarse, lo cual conlleva un alto costo para las instituciones de salud que patrocinan su entrenamiento y especialmente tienen que dejar su área de trabajo, donde generalmente es escaso el recurso humano para la atención de la comunidad. El entrenamiento incluye el aprendizaje de la técnica por medio de la revisión de videos ilustrativos no solo de la técnica en mención, sino de consejería, posteriormente se pasa a entrenamiento en modelos inanimados, como modelos pélvicos, diseñados para tal fin, en los cuales el médico puede practicar cuantas veces quiera la técnica de AQV postparto y de intervalo, incluyendo en esta última el uso de manipulador uterino. Cuando se haya adquirido destreza, se inician las prácticas con pacientes.

Aunque no podemos comparar estadísticamente esta técnica con la convencional, pues no la realizamos en nuestra institución desde la creación de la Unidad de Planificación Familiar, si podemos decir que los costos para la institución se disminuyen grandemente al compa- rar ésta, con técnicas que requieren anestesia general o regional, mayor número de suturas y de estancia hospitalaria. El resultado estético para la paciente es indistinguible del resultado de la laparoscopia, ya que la incisión utilizada es muy similar. El dolor postoperatorio tiene que ser menor pues son incisiones pequeñas y no se utiliza gas intraabdominal como en laparoscopia, hay poca manipulación intraabdominal y bajo tiempo quirúrgico. La recuperación de la mujer a su vida cotidiana es casi inmediata, gran parte de nuestras pacientes tienen sus hijos solos en la casa y es una gran ventaja el que prácticamente en la primera hora postoperatoria, puedan abandonar la institución.

Otra ventaja de este programa es que a mediano y largo plazo disminuirá no solo los costos sino el índice de ingresos hospitalarios por las consecuencias de gestaciones indeseadas y embarazos de alto riesgo.

Creemos que esta técnica es una opción más, que debemos procurar se conozca en las Universidades donde se forman los médicos, que a lo largo y ancho del país ejercerán su profesión y verán limitada su práctica al no disponer de entrenamiento en AQV femenina realizada con recursos mínimos como es el caso de la técnica mostrada, mientras las nuevas legislaciones de seguridad social lo comprometen en forma importante y prioritaria con estas actividades básicas en la atención integral de la mujer.

\section{BIBLIOGRAFIA}

1. Gómez PI., Ruiz N., Pulido J. Mortalidad Materna en el Instituto Materno Infantil de Santafé de Bogotá, D.C. 1985-1989. Rev. Col. Obst. y Ginecol. 1993; 44(1):39-47.

2. Resolución No. 08514 del 27 de Junio de 1984. Ministerio de Salud de Colombia.

3. Artículo 15 de la Constitución Política de Colombia.

4. Dueñas O., Gelvez I., Pacheco C., Gómez PI y Cols. Consejería en la Unidad de Planificación Familiar del Instituto Materno Infantil de Bogotá, D.C. Monografia. Universidad Nacional de Colombia. 1992.
5. Gómez PI. Experiencia de la Unidad Planificación Familiar del Instituto Materno Infantil de Santafé de Bogotá (1990-1993), en Libro: Controversias en Ginecología, Obstetricia y Perinatología. Universidad Nacional de Colombia. Ed. Lerner, 1994.

6. Gómez PI. Planificación Familiar. Memorias V Jornada de Ginecología y Obstetricia, Bogotá, 1989; 93-108.

7. Gómez PI. Manual de Planificación Familiar para Médicos generales. Universidad Nacional de Colombia. 1996.

8. Peterson HB. La efectividad a largo plazo de la esterilización tubárica. Boletín Médico de IPPF. 1996; 30(5): 3-4.

\section{PUBLICACIONES}

Informamos a nuestros lectores que en la oficina de la Revista Colombiana de Obstetricia y Ginecología se encuentran a disposición para consulta las siguientes revistas:

- International Journal of Gynecology \& Obstetrics

- Journal Brasilero de Ginecología

- Anales Chirurgiae et Gynaecologiae

- World Health Organization - Challenges in Reproductive Helth research

- Women's groups. NGOS and Safe Motherhood

- Revista Ecuatoriana de Ginecología y Obstetricia

- International Family Planning Perspectives

- Toko Ginecología Práctica

- Manual de Administración en Planificación Familiar

- Revista Obstetricia y Ginecología de Venezuela

- Revista Centroamericana de Obstetricia y Ginecología 